\title{
Electromagnetic Form Factors of $\Sigma$ Hyperons
}

\author{
Muzaffar Irshad 1,2,*(D), Dong Liu 1,2,3,4,*iD, Xiaorong Zhou $1,2, * \mathbb{D}$ and Guangshun Huang $1,2, * \mathbb{D}$ \\ 1 Department of Modern Physics, University of Science and Technology of China, Hefei 230026, China \\ 2 State Key Laboratory of Particle Detection and Electronics, Hefei 230026, China \\ 3 Helmholtz Institute Mainz, D-55099 Mainz, Germany \\ 4 GSI Helmholtzcentre for Heavy Ion Research GmbH, D-64291 Darmstadt, Germany \\ * Correspondence: subhani09@ustc.edu.cn (M.I.); dliu13@ustc.edu.cn (D.L.); zxrong@ustc.edu.cn (X.Z.); \\ hgs@ustc.edu.cn (G.H.)
}

\begin{abstract}
Electromagnetic form factors (EMFFs) are fundamental observable of baryons that intimately related to their internal structure and dynamics, where the EMFFs of hyperons provide valuable insight into the behavior of the strangeness. The EMFFs of hyperons can also help to understand those of nucleons as they are connected with the flavor SU(3) symmetry. The EMFFs of nucleons can be measured in both spacelike and timelike regions. However, it is difficult to probe the EMFFs of hyperons in spacelike region due to the unstable nature of hyperons. By means of electron-positron annihilation, the EMFFs of hyperons in timelike region is accessible via the production of hyperon-antihyperon pair. The timelike EMFFs of the isospin triplet $\Sigma$ hyperons measured at Babar, CLEO-c and BESIII experiments are reviewed in this paper. Besides, the relevant theoretical discussion based on the experimental results are also presented.
\end{abstract}

Keywords: hyperon structure; form factors; production threshold; annihilation; hyperon

Citation: Irshad, M.; Liu, D.; Zhou, X.; Huang, G. Electromagnetic Form Factors of $\Sigma$ Hyperons. Symmetry 2022, 14, 69. https://doi.org/ 10.3390/sym 14010069

Academic Editors: Monica Bertani, Simone Pacetti and Alessio Mangoni

Received: 1 December 2021

Accepted: 2 January 2022

Published: 4 January 2022

Publisher's Note: MDPI stays neutral with regard to jurisdictional claims in published maps and institutional affiliations.

Copyright: (c) 2022 by the authors. Licensee MDPI, Basel, Switzerland. This article is an open access article distributed under the terms and conditions of the Creative Commons Attribution (CC BY) license (https:// creativecommons.org/licenses/by/ $4.0 /)$.

\section{Introduction}

In the mid-nineteenth century, nucleons, which make up a large portion of the observable matter in the universe, were discovered to be non-pointlike particles. Despite a century of intensive investigation, numerous characteristics of the nucleons remain unsolved, such as their size, spin, and intrinsic structure [1]. In particle physics, the concept of symmetry is a powerful tool to reveal the nature of particles, as it can help one to understand the unknown parts with known ones. In the study of baryons, the concept is also adopted since they are connected by the flavor SU(3) symmetry. One way of probing the inner structure of nucleons is to further extend our knowledge by studying the SU(3) baryon octet partners, often known as hyperons. Electromagnetic form factors (EMFFs), electric $\left(G_{E}\right)$ and magnetic $\left(G_{M}\right)$ of hadrons are essential physical quantities for exploring the internal structure of hadrons and probing the strong interaction in the perturbative and non-perturbative regimes [2-5]. These observables are assumed to be analytic functions of square momentum transfer, $s=q^{2}$, of the virtual photon, and at large momentum transfer $s$, it provides a valuable insight into their quark-gluon structure.

Investigation on nucleon form factors (FFs), particularly effective form factors (EFFs), has been widely explored in both timelike $(s>0)$ [6-17] and spacelike $(s<0)$ [18-22] regions in recent decades, for example, in the electron-hadron elastic scattering, $e^{+} e^{-}$ annihilation reactions. As a result, our understanding on the EMFFs of nucleon has significantly improved. Further insight into the structure of nucleons can also be gained with their SU(3) partners, for example by studying $\Sigma$ hyperons which contain one strange quark [23-25]. Unlike nucleons, it is rather hard to investigate the cross sections and EMFFs of $\Sigma$ hyperons in spacelike region, since hyperons are unstable and cannot be targeted. Instead, $e^{+} e^{-}$annihilation into a $\Sigma \bar{\Sigma}$ pair provides a unique opportunity to explore the electromagnetic (EM) feature of $\Sigma$ hyperons in the timelike region. Thus, the timelike FFs 
appear to be a diagnostic tool to explore the hyperon structure at the production threshold and in the high-s region, where pQCD effects are likely to predominate.

Various experimental and theoretical efforts demonstrate that hyperon studies have advantages over nucleon studies, particularly the self-analyzing nature of hyperon's weak decay, which can be exploited to determine the polarization and spin-correlation parameters for $e^{+} e^{-} \rightarrow \Sigma \bar{\Sigma}$ processes. Those observables can be used to figure out not only bulk properties such as the production cross section and EMFFs, but also the magnitude and relative phase between the $G_{E}(s)$ and $G_{M}(s)$ FFs. As basic quantities of the strong interaction, the EMFFs of hyperon can be predicated using the chiral perturbation theory (ChPT) [26,27], lattice QCD [28], light cone sum rule (LCSR) [29], and unitary and analytic models [3]. The vector meson dominance (VMD) model is now a very successful technique, since it can concurrently characterize EFFs and the ratio $\left|G_{E}(s) / G_{M}(s)\right|$ of $\Sigma$ hyperon. In addition, EMFFs were also parameterized using a pQCD [30] inspired model. The interest is further enhanced by measuring the FFs of $\Sigma$ hyperon at large-s as it provides a sensitive look into possible short-range correlation among the quarks. In the referenced paper [31-33], Jaffe and Wilczek have emphasized the various characteristics of diquark correlations in low-energy QCD dynamics. They claim that the difference in the non-strange quark configuration between the $\Lambda$ and $\Sigma$ isospin triplets makes them a perfect test-bed to study the diquark correlations.

In this review, we mainly focus on the reactions involving $\Sigma$ hyperon-antihyperon pair production final states. The structure of the review is organized as follows. The underlying formalism of the $\Sigma$ hyperon form factor is described in Section 2. The experimental techniques as well as existing data associated with the $\Sigma$ hyperon EMFFs are discussed in Section 3. The theoretical efforts involved in interpreting those hyperon EMFFs results are described in Section 4. A summary and prospects are presented in Section 5.

\section{Formalism for $e^{+} e^{-} \rightarrow \Sigma \bar{\Sigma}$}

The Born cross section of the $e^{+} e^{-} \rightarrow B \bar{B}$ process is formularized in Ref. [34], where $B$ is a spin-1/2 baryon. Assuming one-photon exchange, the differential cross section of the $\Sigma$ hyperon pairs production can be parameterized in terms of EMFFs, $G_{E}$ and $G_{M}$, as

$$
\frac{d \sigma_{\Sigma \Sigma}(s)}{d \Omega}=\frac{\alpha^{2} \beta C}{4 s}\left[\left|G_{M}(s)\right|^{2}\left(1+\cos ^{2} \theta\right)+\frac{2 m_{\Sigma}^{2}}{s}\left|G_{E}(s)\right|^{2} \sin ^{2} \theta\right] .
$$

Integration over angular terms in Equation (1) gives the total cross section of the reaction $e^{+} e^{-} \rightarrow \Sigma \bar{\Sigma}$ as

$$
\sigma_{e^{+} e^{-} \rightarrow \Sigma \bar{\Sigma}}^{\mathrm{B}}(s)=\frac{4 \pi \alpha^{2} \beta C}{3 s}\left[\left|G_{M}(s)\right|^{2}+\frac{2 m_{\Sigma}^{2}}{s}\left|G_{E}(s)\right|^{2}\right],
$$

where $\alpha$ is the fine-structure constant, $s$ is the square momentum transfer, which is equal to the square of center-of-mass (c.m.) energy, $\beta=\sqrt{1-4 m_{\Sigma}^{2} / s}$ is the velocity of the $\Sigma$ hyperon, $m_{\Sigma}$ is the $\Sigma$ mass, $\theta$ is the polar angle of the outgoing $\Sigma$ hyperon in the $e^{+} e^{-}$c.m. frame, and $C$ is the coulomb enhancement factor. The factor $C$ is equal to unity for the neutral $\Sigma$ hyperon pair and $C=\zeta /\left(1-e^{-\zeta}\right)$ with $\zeta=\pi \alpha \sqrt{1-\beta^{2}} / \beta$ for charged $\Sigma$ hyperon pairs [35,36]. From Equation (2), one can expect that the cross section of $e^{+} e^{-} \rightarrow \Sigma \bar{\Sigma}$ is nonzero at the production threshold for charged hyperon pairs and then grows with increasing $\beta$, while it should disappear at the threshold for neutral hyperon pairs.

The magnitude of the effective form factor $\left|G_{\text {eff }}\right|$ can be defined by the combination of $\left|G_{E}(s)\right|$ and $\left|G_{M}(s)\right|[13]$ as:

$$
\left|G_{\text {eff }}(s)\right|=\sqrt{\frac{\left|G_{M}(s)\right|^{2}+\frac{2 m_{\Sigma}^{2}}{s}\left|G_{E}(s)\right|^{2}}{1+\frac{2 m_{\Sigma}^{2}}{s}}}=\sqrt{\frac{\sigma_{e^{+} e^{-} \rightarrow \Sigma \bar{\Sigma}}^{B}(s)}{\frac{4 \pi \alpha^{2} \beta C}{3 s}\left(1+\frac{2 m_{\Sigma}^{2}}{s}\right)}} .
$$




\section{Experiment Results}

\section{1. $e^{+} e^{-} \rightarrow \gamma_{I S R} \Sigma^{0} \bar{\Sigma}^{0}$ at BaBar}

Initial-state-radiation (ISR) technique can be effectively exploited to study physics in $e^{+} e^{-}$annihilation at high luminosity experiments, such as the B-factory at PEP-II. Under the ISR program at BaBar, the cross section measurement for the $e^{+} e^{-} \rightarrow \Sigma^{0} \bar{\Sigma}^{0}$ reaction has been performed by means of the ISR technique [13] from threshold to $3.000 \mathrm{GeV}$. A full reconstruction method has been employed for selecting the $e^{+} e^{-} \rightarrow \Sigma^{0} \bar{\Sigma}^{0} \rightarrow \gamma \gamma p \bar{p} \pi^{+} \pi^{-}$ signal events. The $\Sigma^{0}\left(\bar{\Sigma}^{0}\right)$ particle is reconstructed from $\gamma \Lambda$ decay mode with $\Lambda \rightarrow p \pi$. The cross section for the reaction $e^{+} e^{-} \rightarrow \Sigma^{0} \bar{\Sigma}^{0}$ is then computed in $\Sigma^{0} \bar{\Sigma}^{0}$ mass spectrum and identified about 10 events in the region of 2.385 to $2.600 \mathrm{GeV}$. From the dihyperon mass spectrum $M_{\Sigma^{0} \bar{\Sigma}^{0}}$, BaBar reported the corresponding effective form factor of $\Sigma^{0}$ hyperon and compared it with other dibaryon pairs as shown in Figure 1. The non-zero cross section of $e^{+} e^{-} \rightarrow \Sigma^{0} \bar{\Sigma}^{0}$ reaction near threshold gives a clear sign of the role of Coulomb factor for pairs of neutral hyperons. However, large uncertainties due to small statistics make it difficult to interpret these findings in terms of pQCD.

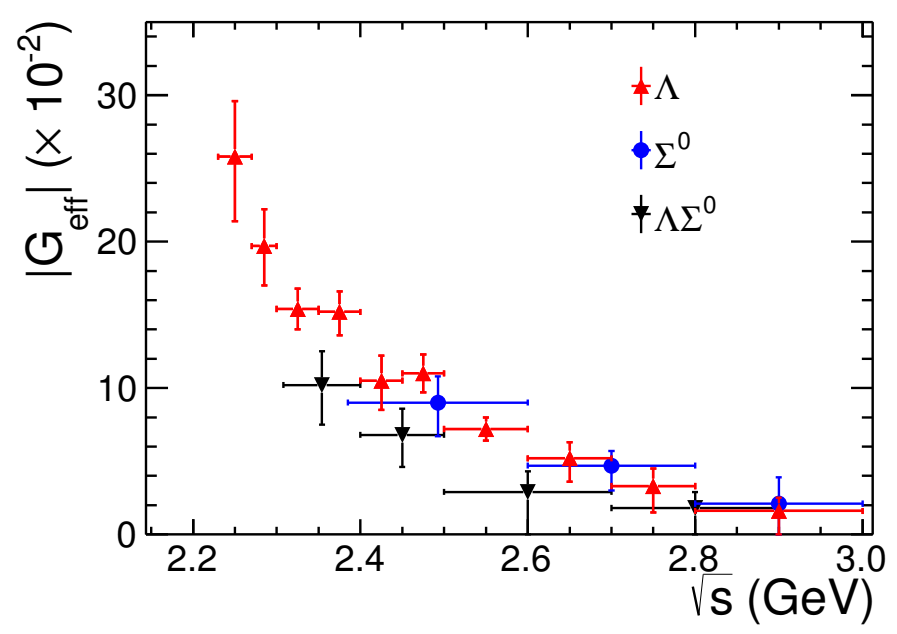

Figure 1. Comparison of effective form factor of $\Sigma^{0}$ to those of other hyperons [13].

\section{2. $e^{+} e^{-} \rightarrow \Sigma \bar{\Sigma}$ at CLEO-c}

As reviewed in previous Section 3.1, the cross section of $e^{+} e^{-}$annihilation into hyperon pairs is predicted to be very small, and only two experimental results of FFs for $\Lambda, \Sigma^{0}$ hyperons exist in the literature with limited statistics. Using $e^{+} e^{-}$annihilation data taken at the CESR collider with the CLEO-c detector, the world's first measurements of the form factors of hyperons with one or multi-strange quark composition, $\Lambda, \Sigma^{0}, \Sigma^{+}, \Xi^{0}, \Xi^{-}$, and $\Omega^{-}$ at $s=14.2$ and $17.4 \mathrm{GeV}^{2}$ were documented in Ref. [37], and the latter with substantially improved precision, presented in Ref. [38]. The analysis is based on the data collected at charmonium resonances such as $\psi(2 S), s=13.7 \mathrm{GeV}^{2}, \psi(3770), s=14.2 \mathrm{GeV}^{2}, \psi(4170)$, $s=17.4 \mathrm{GeV}^{2}$, with integrated luminosities of $\mathcal{L}=48 \mathrm{pb}^{-1}, 805 \mathrm{pb}^{-1}, 586 \mathrm{pb}^{-1}$, respectively. Due to topic limitation, we only emphasized the form factors of $\Sigma$ hyperons. In this analysis $\Sigma$ hyperons are reconstructed by their major decay modes as reported by the Particle Data Group (PDG) [39]: $\Sigma^{+} \rightarrow p \pi^{0}(51.6 \%), \Sigma^{0} \rightarrow \gamma \Lambda$ (100\%) with subsequent decay $\Lambda \rightarrow p \pi^{-}(63.9 \%)$. The charge conjugate modes of $\Sigma$ are included by default.

The hadron pair production at $s=13.7 \mathrm{GeV}^{2}$ is dominated by strong decay of the $\psi(2 S)$ resonance with large cross sections. Consequently, the resulting pair production cross sections are smaller by factors as large as several hundred. Unlike at $\psi(2 S)$, the resonance production of hadron pairs at $\psi(3770)$ and $\psi(4170)$ is negligibly small as predicted by pQCD and the non-resonance EM production of hadron pairs would dominate in this region and can be used to measure the EMFFs. The expectation was well confirmed by the measurements of the FFs of pion, kaon, and proton at $\psi(3770)$ and $\psi(4170)$ [40]. 
Despite this fact, the contributions of resonance decay to hyperon-antihyperon pairs in this measurement are negligibly small at $\psi(3770)$ and $\psi(4170)$, and the observed events can be safely attributed to EM production, i.e., $e^{+} e^{-} \rightarrow \gamma^{*} \rightarrow B \bar{B}$. Thus, at $\psi(3770)$ and $\psi(4170)$ pair production is entirely EM, thereby the measured cross sections are smaller by orders of magnitude [38]. The obtained cross section of $\Sigma$ pairs for $\psi(3770)$ data were $\sigma\left(e^{+} e^{-} \rightarrow\right.$ $\left.\Sigma^{0} \bar{\Sigma}^{0}\right)=(0.48 \pm 0.07 \pm 0.02) \mathrm{pb}$ and $\sigma\left(e^{+} e^{-} \rightarrow \Sigma^{+} \bar{\Sigma}^{-}\right)=(1.02 \pm 0.10 \pm 0.04) \mathrm{pb}$. The cross section results from $\psi(4170)$ data were to be $\sigma\left(e^{+} e^{-} \rightarrow \Sigma^{0} \bar{\Sigma}^{0}\right)=(0.09 \pm 0.04 \pm$ $0.02) \mathrm{pb}$ and $\sigma\left(e^{+} e^{-} \rightarrow \Sigma^{+} \bar{\Sigma}^{-}\right)=(0.23 \pm 0.06 \pm 0.04) \mathrm{pb}$. From these measurements, the pair production cross section of $\Sigma$ hyperon at $\psi(4170)$ is smaller by a factor of 4 to 6 than that at $\psi(3770)$, and the cross sections have much large relative uncertainties. The dramatically changed in the cross sections are due to the fact that the quark counting rules of QCD [41] and the energy dependence. The EM cross sections for baryons generally falls as $1 / s^{5}$, which would lead to a constant ratio, $R=\sigma(3.77 \mathrm{GeV}) / \sigma(4.17 \mathrm{GeV})=2.74$ for all hyperons. In Figure $2 \mathrm{a}$, the ratio varies substantially with the number $n_{S}$ of strange quarks in the hyperon, being $R\left(n_{s}=0\right.$, proton $)=0.5, R\left(n_{s}=1, \Lambda, \Sigma^{0}, \Sigma^{+}\right) \approx 4$, and $R\left(n_{s}=2, \Xi^{-}, \Xi^{0}\right) \approx 10[38]$.
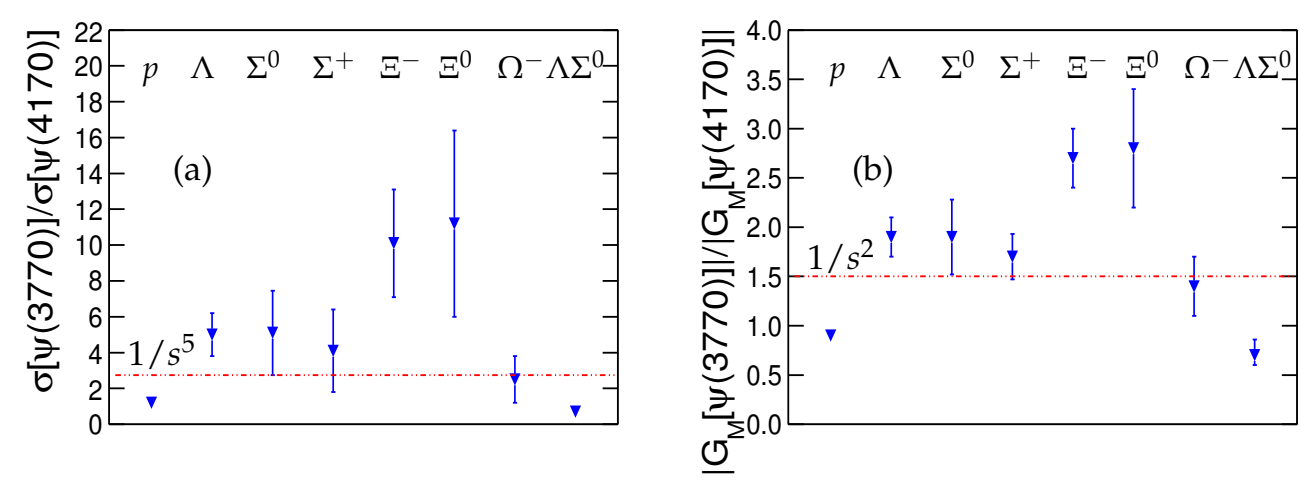

Figure 2. Comparison of baryons pair productions at $\psi(3770)$ and $\psi(4170)$. (a) The cross Section ratios [38] differ from the theoretically predicated ratios of $1 / s^{5}, 2.74$, and shows clear difference for baryons containing different numbers of strange quarks. (b) The ratios of $G_{M}$ [38] also differ from the prediction of $1 / s^{2}, 1.5$.

The EM production data was analyzed in terms of the traditional electric $G_{E}(s)$ and magnetic $G_{M}(s)$ FFs as a function of $s$, see in Equation (1). In analyses of data owing to limited statistics, it is not possible to determine the ratio $\left|G_{E}(s) / G_{M}(s)\right|$ of hyperons by analyzing the angular distribution of the cross sections, and data are often analyzed for two extreme values, $\left|G_{E}(s) / G_{M}(s)\right|=0$ and 1 . The timelike form factors $G_{M}(s)$ with the assumption $G_{E}(s)=0$ at $s=14.2 \mathrm{GeV}^{2}$ were determined to be $G_{M}^{\Sigma^{0}}(s)=(1.01 \pm 0.07 \pm 0.02) \times 10^{2}$ and $G_{M}^{\Sigma^{+}}(s)=(1.47 \pm 0.07 \pm 0.03) \times 10^{2}$, and for data at $s=17.4 \mathrm{GeV}^{2}$ to be $G_{M}^{\Sigma^{0}}(s)=$ $(0.47 \pm 0.09 \pm 0.04) \times 10^{2}$ and $G_{M}^{\Sigma^{+}}(s)=(0.75 \pm 0.09 \pm 0.06) \times 10^{2}$, respectively. The results corresponding to $G_{E}(s)=G_{M}(s)$ assumption were between $8 \%$ and $18 \%$ smaller than those obtained by $G_{E}(s)=0$ assumption [37,38]. The ratios of $G_{M}$ at $\psi(3770)$ and $\psi(4170)$ are shown in Figure $2 \mathrm{~b}$ deviated from constant ratio as predicted by QCD, $1 / s_{\psi(3770)}^{2}: 1 / s_{\psi(4170)}^{2}=1.5$ for baryon containing 0,1 and 2 strange quarks. Another important finding concerns the electromagnetic production cross section of $\Sigma^{0}$, which is significantly suppressed compared to those of $\Lambda$, and its explanation in terms of diquark correlation. The ratios $\sigma(\Lambda) / \sigma\left(\Sigma^{0}\right)=2.46 \pm 0.46$ at $\psi(3770)$, and $\sigma(\Lambda) / \sigma\left(\Sigma^{0}\right)=2.56 \pm 1.40$ at $\psi(4170)$ were strong independent confirmation of diquark correlations in the structure of $\Lambda$ and $\Sigma^{0}$ hyperons.

\section{3. $e^{+} e^{-} \rightarrow \Sigma \bar{\Sigma}$ at BESIII}

Recently, using scan energy data, the BESIII experiment investigated the $e^{+} e^{-} \rightarrow$ $\Sigma^{ \pm} \bar{\Sigma}^{\mp}$ and $e^{+} e^{-} \rightarrow \Sigma^{0} \bar{\Sigma}^{0}$ reactions from 2.3864 to $3.0200 \mathrm{GeV}$ and determined the timelike 
EMFFs of $\Sigma$ hyperon with very high precision [42,43]. The charged channels $e^{+} e^{-} \rightarrow \Sigma^{ \pm} \bar{\Sigma}^{\mp}$ were reconstructed for the first time in the continuum (off-resonance) region. Born cross sections of $\Sigma^{ \pm} \bar{\Sigma}^{\mp}$ pair productions, effective form factors $\left|G_{\text {eff }}\right|$ of $\Sigma^{+}$and $\Sigma^{-}$, the ratios of $\Sigma^{+}$electric and magnetic FFs $\left|G_{E} / G_{M}\right|$, were reported [42]. For c.m.energies near threshold, a novel method was used to reconstruct the neutral channel $e^{+} e^{-} \rightarrow \Sigma^{0} \bar{\Sigma}^{0}$ whereas a single-hyperon-tag method was applied for c.m. energies between 2.5000 and $3.0200 \mathrm{GeV}$. Born cross sections are measured with significantly improved precision [43] to those of BaBar [13]. In addition, the $\left|G_{\text {eff }}\right|$ of $\Sigma^{0}$ was also reported. These findings provide precise experimental input to test the several theoretical calculations and a deep understanding of the $\Sigma$ hyperons structure.

The precision has significantly improved in the analysis of the $e^{+} e^{-} \rightarrow \Sigma^{+} \bar{\Sigma}^{-}$reaction by reconstructing all four dominated final states according to PDG [39], $p \pi^{0} \bar{p} \pi^{0}, n \pi^{+} \bar{p} \pi^{0}$, $p \pi^{0} \bar{n} \pi^{-}$and $n \pi^{+} \bar{n} \pi^{-}$, respectively. The $p \pi^{0} \bar{p} \pi^{0}$ and $n \pi^{+} \bar{p} \pi^{0}$ final state topologies have been classified as category A, and a single-tag baryon technique has been imposed to only detect the $\Sigma^{-} \rightarrow \bar{p} \pi^{0}$ prong. Besides, the $p \pi^{0} \bar{n} \pi^{-}$and $n \pi^{+} \bar{n} \pi^{-}$final states are classified as category B, and analyzed with one-particle missing technique [42]. The determined cross sections for $e^{+} e^{-} \rightarrow \Sigma^{ \pm} \bar{\Sigma}^{\mp}$ reactions are shown in Figure 3 from 2.3864 to $3.0200 \mathrm{GeV}$.

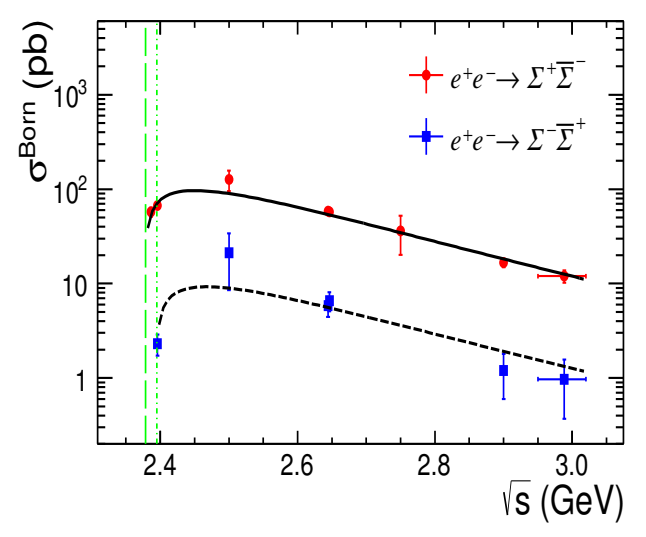

Figure 3. The cross section lineshapes for $e^{+} e^{-} \rightarrow \Sigma^{+} \bar{\Sigma}^{-}$reactions (circles) and $e^{+} e^{-} \rightarrow \Sigma^{-} \bar{\Sigma}^{+}$ (squares) [42]. The solid and dashed smooth lines are the pQCD fit for $e^{+} e^{-} \rightarrow \Sigma^{+} \bar{\Sigma}^{-}$and $e^{+} e^{-} \rightarrow$ $\Sigma^{-} \bar{\Sigma}^{+}$, respectively. The vertical lines denoted their production thresholds.

Near production threshold of $\Sigma^{ \pm} \bar{\Sigma}^{\mp}$ pairs, the cross section were observed to be $\left(58.2 \pm 5.9_{-2.6}^{+2.8}\right) \mathrm{pb}$ and $(2.3 \pm 0.5 \pm 0.3) \mathrm{pb}$, which disagrees with the pointlike expectations close to threshold of $848\left(m_{p} / m_{B}\right)^{2} \mathrm{pb}$. The cross section lineshapes presented in Figure 3 for $e^{+} e^{-} \rightarrow \Sigma^{+} \bar{\Sigma}^{-}$and $e^{+} e^{-} \rightarrow \Sigma^{-} \bar{\Sigma}^{+}$are well-described by pQCD-motivated functions. The ratio of the $\sigma^{\text {Born }}\left(e^{+} e^{-} \rightarrow \Sigma^{+} \bar{\Sigma}^{-}\right)$to $\sigma^{\text {Born }}\left(e^{+} e^{-} \rightarrow \Sigma^{-} \bar{\Sigma}^{+}\right)$was found to be $9.7 \pm 1.3$, which is inconsistent with predictions from several models [3,26,27,31-33,44,45]. The EFF is proportional to the square root of the cross section, and the observed ratio of $\left|G_{\text {eff }}^{\Sigma^{+}}(s)\right| /\left|G_{\text {eff }}^{\Sigma^{-}}(s)\right|$ was found to be consistent with 3 , which is the ratio of the incoherent sum of the squared charges of valence quarks in $\Sigma^{+}$and $\Sigma^{-}$baryons, $\Sigma_{q} Q_{q}^{2}$, with $q=u, d$, s. Furthermore, the EMFF ratio $\left|G_{E}(s) / G_{M}(s)\right|$ of the $\Sigma^{+}$was reported through an angular analysis at three high-statistics energy points, 2.3960,2.6444, and $2.6464 \mathrm{GeV}$ for $e^{+} e^{-} \rightarrow$ $\Sigma^{+} \bar{\Sigma}^{-}$. The study based on the polar angular distribution of $\Sigma^{+}$, the ratio $\left|G_{E}(s) / G_{M}(s)\right|$ of the $\Sigma^{+}$baryon was determined to be $\left|G_{E}(s) / G_{M}(s)\right|=1.83 \pm 0.26$ near threshold as shown in Figure 4, which is significantly higher than 1 [42]. The ratio $\left|G_{E}(s) / G_{M}(s)\right|$ of the $\Sigma^{+}$shows similar features to that of other baryons like proton $[9,14,15], \Lambda[46,47]$, and $\Lambda_{c}$ [36], which are all bigger than 1 within uncertainties near production threshold and consistent with 1 at higher-s. 

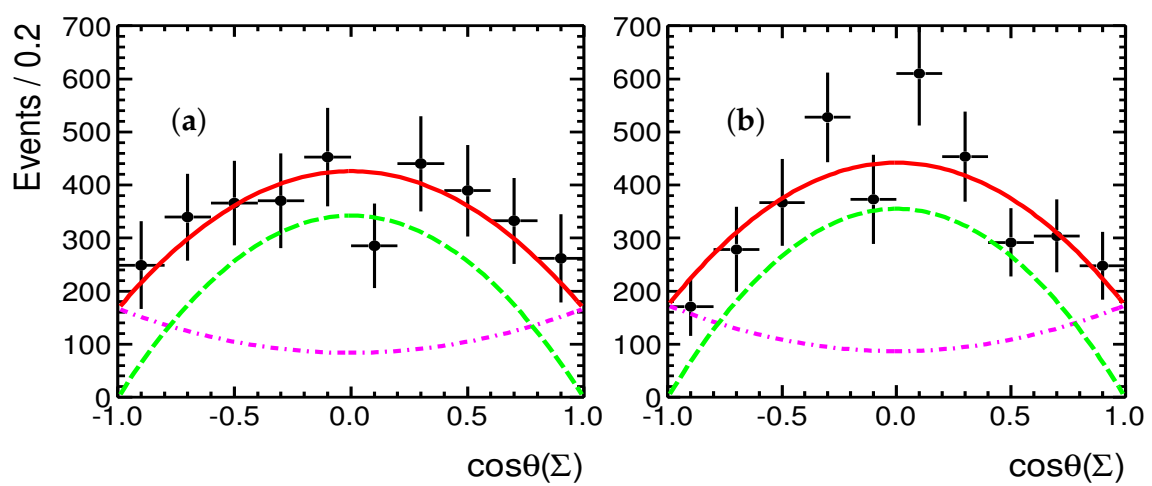

Figure 4. The polar angular distributions at c.m. energy $2.3960 \mathrm{GeV}$ for (a) category A (b) category B for $e^{+} e^{-} \rightarrow \Sigma^{+} \bar{\Sigma}^{-}$reaction. The dots with error bars are from data, solid curves are the fit results, and the contributions from $G_{E}$ and $G_{M}$ are represented by dashed and dotted curves [42].

Further deep insight to $\Sigma$ hyperon structure, the reaction channel $e^{+} e^{-} \rightarrow \Sigma^{0} \bar{\Sigma}^{0}$ was investigated at $\sqrt{s}=2.3864 \mathrm{GeV}$ and $2.3960 \mathrm{GeV}$. The signal were reconstructed with the final state topology $\gamma \gamma p \bar{p} \pi^{+} \pi^{-}$. Because of low momentum final state particles near threshold and low photon detection efficiency from the signal, this would eventually lead to a higher multiplicity of background photons. As a result, only soft pions and the secondary products of antiproton interactions were reconstructed near production threshold as method described in Refs. [46,47]. A single-tag baryon method was used to reconstruct $\Sigma^{0}$ in the $\gamma \Lambda$ decay mode with $\Lambda \rightarrow p \pi^{-}$at higher c.m. energies from 2.5000 to $3.0200 \mathrm{GeV}$. The charge conjugation mode of $\Sigma^{0}$-prong is implied by default in the single-tag method.

Born cross sections and EFFs are presented in Figure 5. Since at $2.3864 \mathrm{GeV}$ owing to limited statistics thereby no significant signal was observed near threshold and an upper limit (U.L.) on the observed cross section at the $90 \%$ C.L. was reported $\sigma_{\text {U.L. }}\left(e^{+} e^{-} \rightarrow\right.$ $\left.\Sigma^{0} \bar{\Sigma}^{0}\right)<42.4 \mathrm{pb}$. In conclusion, no threshold effect was seen for $e^{+} e^{-} \rightarrow \Sigma^{0} \bar{\Sigma}^{0}$ reaction, as in common with the behavior observed in an earlier analysis of $e^{+} e^{-} \rightarrow \Sigma^{ \pm} \bar{\Sigma}^{\mp}$ [42]. From Figure 5, the cross section lineshape for $e^{+} e^{-} \rightarrow \Sigma^{0} \bar{\Sigma}^{0}$ is well described with a pQCDmotivated function. Moreover, the significantly suppressed cross section of $e^{+} e^{-} \rightarrow \Sigma^{0} \bar{\Sigma}^{0}$ compared to those of $e^{+} e^{-} \rightarrow \Lambda \bar{\Lambda}$ could provide experimental input to test the diquark correlation model. An asymmetry of the EFFs of $\Sigma$ isospin triplet was observed, which is consistent with their incoherent sum of squared charges of valence quarks [42].
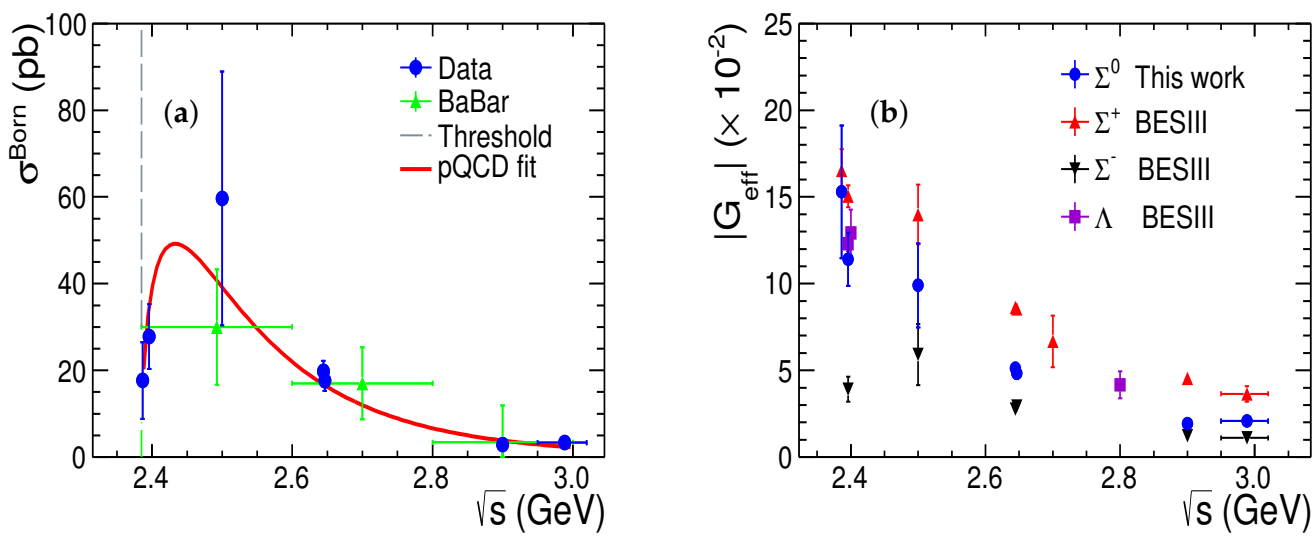

Figure 5. (a) Comparison plot of the cross sections for $e^{+} e^{-} \rightarrow \Sigma^{0} \bar{\Sigma}^{0}$ reaction. The triangles in green are results from BaBar [13]. The solid line in red shows the PQCD fit. (b) Comparison plot of the EFFs of hyperons from BESIII results $[42,46,47]$. 


\section{Theoretical Intepretations}

Many surprising aspects of the reaction $e^{+} e^{-} \rightarrow \Sigma \bar{\Sigma}$ near production thresholds have been seen experimentally. These unusual $\Sigma \bar{\Sigma}$ pair production threshold effects have been extensively addressed in the literature, where they have been interpreted as final state interactions [5], bound states or unobserved meson resonances [48], or possibly attractive and repulsive Coulomb forces between quarks [49,50].

In the Jülich meson-exchange model the hyperon-antihyperon production is considered within a coupled-channel approach. This enables the impact of the final state $(\Sigma \bar{\Sigma})$ interactions to be taken into account rigorously. They emphasized that the final state Coulomb correction, so-called enhancement factor, $C$, to the cross section should be considered in case of pointlike charged hyperon pair production. However, it has been noted in the Refs. [49-51] that a quite bigger in amount and energy interval, threshold enhancement factor due to strong interactions is predicted in the case of heavy quark-pair creation by $e^{+} e^{-}$annihilation. Low-s gluon exchange should be introduced in the cross section as a factor comparable to the Coulomb correction, with $\frac{4}{3} \alpha_{S}(s)$ instead of $\alpha$. With regards to the processes $e^{+} e^{-} \rightarrow \Sigma^{ \pm} \bar{\Sigma}^{\mp}, \Sigma^{0} \bar{\Sigma}^{0}$, a delicate interplay between these channels was observed [42,43], especially near production threshold, and thought to be caused by the $\Sigma \bar{\Sigma}$ final state interaction [5]. As documented in Reference [5], the cross sections of $e^{+} e^{-} \rightarrow \Sigma \bar{\Sigma}$ exhibit a weak energy dependence that is more modest than the pQCD parameterization [30]. In addition, prediction based on Babar measurement for $e^{+} e^{-} \rightarrow \Sigma^{0} \bar{\Sigma}^{0}$ [13] reaction shows a different energy dependence comparing to $e^{+} e^{-} \rightarrow \Sigma^{ \pm} \bar{\Sigma}^{\mp}$ channels. This notable feature can also be seen in the result of $G_{\text {eff }}$ in Figure 7 of Ref. [5], namely a pronounced structure at the $\Sigma^{-} \bar{\Sigma}^{+}$threshold. Nevertheless, the most recent measurement on $e^{+} e^{-} \rightarrow \Sigma^{0} \bar{\Sigma}^{0}$ reaction investigated by BESIII [43] is inconsistent with the expectation based on the hyperon-antihyperon potential model.

Very recently measured EFFs $\left|G_{\text {eff }}\right|$ of $\Sigma^{+}$and $\Sigma^{-}$[42] were used to determine the parameters of VMD model [30] by fitting them to the BESIII experimental data, where contributions from $\rho, \omega$ and $\phi$ mesons are taken into consideration. Theoretical results documented in Ref. [5] with $\left|G_{\text {eff }}\right|$ in Figure 7 and the ratio $\left|G_{\text {eff }}^{\Sigma^{+}}(s)\right| /\left|G_{\text {eff }}^{\Sigma^{-}}(s)\right|$ in Figure 8 can provide a satisfactory description for the BESIII experimental data [42]. Moreover, the ratios $\left|G_{E}(s) / G_{M}(s)\right|$ for $\Sigma^{+}$and $\Sigma^{-}$were found to be one at mass threshold of hyperon pairs, while for c.m. energies between 2.4000 and $3.0000 \mathrm{GeV}$, the ratio is slowly decreasing for $\Sigma^{0}$, and almost flat for $\Sigma^{-}$. Theoretical results are unable to adequately explain the experimental data well, since the determined ratio is greater than 1 within the uncertainties near threshold [42], implying that there should be other contributions around that energy region.

\section{Summary and Prospect}

In summary, we review the recent experimental studies on timelike EMFFs of $\Sigma$ hyperons by means of hyperon pair production close to their thresholds and above the open charm threshold reported by various experiments. These EMFFs results on $\Sigma$-isospin triplets provide experimental inputs for testing various theoretical models such as hyperonantihyperon potential models, ChPT, lattice QCD, light cone sum rule, VMD model, pQCD inspired models, and diquark correlation model and expanding our knowledge of the $\Sigma$ structure. Besides, the recent theoretical activities are also reviewed in this paper to investigate the EMFFs of $\Sigma$ hyperons based on recent measurements and the required parameters for various models were determined. In view of the facts, the energy dependence of the cross sections for all three reactions $e^{+} e^{-} \rightarrow \Sigma^{ \pm} \bar{\Sigma}^{\mp}, \Sigma^{0} \bar{\Sigma}^{0}$ at c.m. energies near production threshold is not very clear. Therefore, Using $e^{+} e^{-} \rightarrow \Sigma^{0} \bar{\Sigma}^{0}$ results as input would be an exciting way to perform further theoretical calculations and establish reliable predictions for various models. More experimental results by studying the hyperon EMFFs will be made with larger data sets in $e^{+} e^{-}$annihilation at BESIII [52], and the proposed Super Tau-Charm Factory project in China [53] and Russia [54], and the upcoming future facility PANDA in $p \bar{p}$ annihilation at FAIR [55]. 
Author Contributions: Conceptualization, M.I., D.L. and G.H.; Formal analysis, M.I.; Funding acquisition, G.H.; Investigation, M.I.; Methodology, M.I.; Supervision, X.Z., D.L. and G.H.; Validation, X.Z.; Writing-original draft, M.I.; Writing—review \& editing, M.I. and G.H. All authors have read and agreed to the published a version of the manuscript.

Funding: This work is supported in part by National Key Basic Research Program of China under Contract No. 2020YFA0406403; National Natural Science Foundation of China (NSFC) under Contracts Nos. 12035013, 12061131003, 11335008, 12105276, 11911530140; Joint Large-Scale Scientific Facility Funds of the NSFC and CAS under Contracts No. U1832103.

Institutional Review Board Statement: Not applicable.

Informed Consent Statement: Not applicable.

Data Availability Statement: Not applicable.

Acknowledgments: We thank the anonymous guest editors: Monica Bertani, Simone Pacetti and Alessio Mangoni for their kind invitations and help.

Conflicts of Interest: The authors declare no conflict of interest.

\section{References}

1. Miller, G.A. Charge densities of the neutron and proton. Phys. Lett. B 2007, 99, 112001. [CrossRef] [PubMed]

2. Yang, Y.; Chen, D.Y.; Lu, Z. Electromagnetic form factors of $\Lambda$ hyperon in the vector meson dominance model. Phys. Rev. D 2019, 100, 073007. [CrossRef]

3. Ramalho, G.; Pena, M.T.; Tsushima, K. Hyperon electromagnetic timelike elastic form factors at large $q^{2}$. Phys. Rev. D 2020, 101, 014014. [CrossRef]

4. Yang, M.; Wang, P. Electromagnetic form factors of octet baryons with the nonlocal chiral effective theory. Phys. Rev. D 2020, 102, 056024. [CrossRef]

5. Haidenbauer, J.; Meißner, U.-G.; Dai, L.-Y. Hyperon electromagnetic form factors in the timelike region. Phys. Rev. D 2021, 103, 014028. [CrossRef]

6. Akhmetshin, R.R.; Amirkhanov, A.N.; Anisenkov, A.V.; Aulchenko, V.M.; Banzarov, V.S.; Bashtovoy, N.S.; Berkaev, D.E.; Bondar, A.E.; Bragin, A.V.; Eidelman, S.I.; et al. Study of the process $e^{+} e^{-} \rightarrow p \bar{p}$ in the c.m. energy range from threshold to $2 \mathrm{GeV}$ with the CMD-3 detector. Phys. Lett. B 2016, 759, 634. [CrossRef]

7. Andreotti, M.; Bagnasco, S.; Baldini, W.; Bettoni, D.; Borreani, G.; Buzzo, A.; Calabrese, R.; Cester, R.; Cibinetto, G.; Dalpiaz, P.; et al. Measurements of the magnetic form-factor of the proton for timelike momentum transfers. Phys. Lett. B 2003, 559, 20. [CrossRef]

8. Antonelli, A.; Baldini, R.; Benasi, P.; Bertani, M.; Biagini, M.E.; Bidoli, V.; Bini, C.; Bressani, T.; Calabrese, R.; Cardarelli, R.; et al. The first measurement of the neutron electromagnetic form-factors in the timelike region. Nucl. Phys. B 1998, 517, 3. [CrossRef]

9. Ablikim, M.; Achasov, M.N.; Adlarson, P.; Ahmed, S.; Albrecht, M.; Alekseev, M.; Amoroso, A.; An, F.F.; An, Q.; Bai, Y.; et al. Measurement of proton electromagnetic form factors in $e^{+} e^{-} \rightarrow p \bar{p}$ in the energy region 2.00-3.08 GeV. Phys. Rev. Lett. 2020, 124, 042001. [CrossRef] [PubMed]

10. Bardin, G.; Burgun, G.; Calabrese, R.; Capon, G.; Carlin, R.; Dalpiaz, P.; Dalpiaz, P.F.; Derré J.; Dosselli, U.; Duclos, J.; et al. Determination of the electric and magnetic form factors of the proton in the timelike region. Nucl. Phys. B 1994, 411, 3. [CrossRef]

11. Bisello, D.; Limentani, S.; Nigro, M.; Pescara, L.; Posocco, M.; Sartori, P.; Augustin, J.E.; Busetto, G.; Cosme, G.; Couchot, F.; et al. A measurement of $e^{+} e^{-} \rightarrow p \bar{p}$ for $(1975 \leq \sqrt{s} \leq 2250)$ MeV. Nucl. Phys. B 1983, 224, 379. [CrossRef]

12. Ambrogiani, M.; Bagnasco, S.; Baldini, W.; Bettoni, D.; Borreani, G.; Buzzo, A.; Calabrese, R.; Cester, R.; Dalpiaz, P.; Fan, X.; et al. Measurements of the magnetic form-factor of the proton in the timelike region at large momentum transfer. Phys. Rev. D 1999, 60, 032002. [CrossRef]

13. Aubert, B.; Bona, M.; Boutigny, D.; Karyotakis, Y.; Lees, J.P.; Poireau, V.; Prudent, X.; Tisserand, V.; Zghiche, A.; Tico, J.G.; et al. Study of $e^{+} e^{-} \rightarrow \Lambda \bar{\Lambda}, \Lambda \bar{\Sigma}^{0}, \Sigma^{0} \bar{\Sigma}^{0}$ using ISR. Phys. Rev. D 2007, 76, 092006. [CrossRef]

14. Lees, J.P.; Poireau, V.; Tisserand, V.; Grauges, E.; Palano, A.; Eigen, G.; Stugu, B.; Brown, D.N.; Kerth, L.T.; Kolomensky, Y.G.; et al. Measurement of the $e^{+} e^{-} \rightarrow p \bar{p}$ cross section in the energy range from 3.0 to $6.5 \mathrm{GeV}$. Phys. Rev. D 2013, 88, 072009. [CrossRef]

15. Lees, J.P.; Poireau, V.; Tisserand, V.; Grauges, E.; Palano, A.; Eigen, G.; Stugu, B.; Brown, D.N.; Kerth, L.T.; Kolomensky, Y.G.; et al. Study of $e^{+} e^{-} \rightarrow p \bar{p}$ via initial-state radiation at BaBar. Phys. Rev. D 2013, 87, 092005. [CrossRef]

16. Ablikim, M.; Achasov, M.N.; Ai, X.C.; Albayrak, O.; Albrecht, M.; Ambrose, D.J.; Amoroso, A.; An, F.F.; An, Q.; Bai, J.Z.; et al. Measurement of the proton form factor by studying $e^{+} e^{-} \rightarrow p \bar{p}$. Phys. Rev. D 2015, 91, 112004. [CrossRef]

17. Armstrong, T.A.; Bettoni, D.; Bharadwaj, V.; Biino, C.; Borreani, G.; Broemmelsiek, D.; Buzzo, A.; Calabrese, R.; Ceccucci, A.; Cester, R.; et al. Measurement of the proton electromagnetic form-factors in the timelike region at 8.9 to $13 \mathrm{GeV}^{2}$. Phys. Rev. Lett. 1993, 70, 1212. [CrossRef] 
18. Passchier, I.; van Buuren, L.D.; Szczerba, D.; Alarcon, R.; Bauer, T.S.; Boersma, D.J.; van den Brand, J.F.J.; Bulten, H.J.; Ent, R.; erro-Luzzi, M.; et al. Spin momentum correlations in quasielastic electron scattering from deuterium. Phys. Rev. Lett. 2002, 88,102302 . [CrossRef]

19. Gayou, O.; Aniol, K.A.; Averett, T.; Benmokhtar, F.; Bertozzi, W.; Bimbot, L.; Brash, E.J.; Calarco, J.R.; Cavata, C.; Chai, Z.; et al. Measurement of $G_{E p} / G_{M p}$ in $\vec{e} p \rightarrow e \vec{p}$ to $Q^{2}=5.6 \mathrm{GeV}^{2} / c^{2}$. Phys. Rev. Lett. 2002, 88, 092301. [CrossRef] [PubMed]

20. Madey, R.; Semenov, A.Y.; Taylor, S.; Plaster, B.; Aghalaryan, A.; Crouse, E.; MacLachlan, G.; Tajima, S.; Tireman, W.; Yan, C.; et al. Measurements of the neutron electric to magnetic form-factor ratio $G_{E n} / G_{M n}$ via ${ }^{2} H\left(\left(\vec{e}, e^{\prime} n\right)^{1} H\right.$ reaction to $Q^{2}=1.45(\mathrm{GeV} / c)^{2}$. Phys. Rev. Lett. 2003, 91, 122002. [CrossRef] [PubMed]

21. Warren, G.; Wesselmann, F.; Zhu, H.; McKee, P.; Savvinov, N.; Zeier, M.; Aghalaryan, A.; Ahmidouch, A.; Arenhövel, H.; Asaturyan, R.; et al. Measurement of the electric form-factor of the neutron at $Q^{2}=0.5$ and $1.0 \mathrm{GeV}^{2} / c^{2}$. Phys. Rev. Lett. 2004, 92, 042301. [CrossRef] [PubMed]

22. Huang, G.; Ferroli, R.B. Probing the internal structure of baryons. Natl. Sci. Rev. 2021, 8, nwab187. [CrossRef]

23. Ramalho, G.; Tsushima, K.; Thomas, A.W. Octet baryon electromagnetic form factors in nuclear medium. J. Phys. G 2013, 40, 015102. [CrossRef]

24. Gross, F.; Ramalho, G.; Tsushima, K. Using baryon octet magnetic moments and masses to fix the pion cloud contribution. Phys. Lett. B 2010, 690, 183. [CrossRef]

25. Eichmann, G.; Sanchis-Alepuz, H.; Williams, R.; Alkofer, R.; Fischer, C.S. Review on the progress in nuclear fission experimental methods and theoretical descriptions. Prog. Part. Nucl. Phys. 2016, 91, 1. [CrossRef]

26. Kubis, B.; Meissner, U.G. Baryon form-factors in chiral perturbation theory. Eur. Phys. J. C 2001, 18, 747. [CrossRef]

27. Geng, L.S.; Camalich, J.M.; Alvarez-Ruso, L.; Vacas, M.J.V. Leading SU(3)-breaking corrections to the baryon magnetic moments in Chiral Perturbation Theory. Phys. Rev. Lett. 2008, 101, 222002. [CrossRef] [PubMed]

28. Green, J.R.; Negele, J.W.; Pochinsky, A.V.; Syritsyn, S.N.; Engelhardt, M.; Krieg, S. Threshold expansion of the three-particle quantization condition. Phys. Rev. D 2014, 90, 074507. [CrossRef]

29. Liu, Y.L.; Huang, M.Q. Electromagnetic form factors of the $\Lambda$ and $\Sigma$ baryons in an alternative baryonic current approach. Phys. Rev. D 2009, 79, 114031. [CrossRef]

30. Brodsky, S.J.; Farrar, G.R. Scaling laws for large momentum transfer processes. Phys. Rev. D 1975, 11, 1309. [CrossRef]

31. Anselmino, M.; Predazzi, E.; Ekelin, S.; Fredriksson, S.; Lichtenberg, D.B. Diquarks. Rev. Mod. Phys. 1993, 65, 1199. [CrossRef]

32. Jaffe, R.L.; Wilczek, F. Diquarks and exotic spectroscopy. Phys. Rev. Lett. 2003, 91, 232003. [CrossRef] [PubMed]

33. Jaffe, R.L. Exotica. Phys. Rep. 2005, 409, 1. [CrossRef]

34. Cabibbo, N.; Gatto, R. Electron Positron Colliding Beam Experiments. Phys. Rev. 1961, 124, 1577. [CrossRef]

35. Brodsky, J.S.; Lebed, F.R. Production of the Smallest QED Atom: True Muonium $\left(\mu^{+} \mu^{-}\right)$. Phys. Rev. Lett. 2009, $102,213401$. [CrossRef]

36. Ablikim, M.; Achasov, M.N.; Ahmed, S.; Albrecht, M.; Alekseev, M.; Amoroso, A.; An, F.F.; An, Q.; Bai, J.Z.; Bai, Y.; et al. Precision measurement of the $e^{+} e^{-} \rightarrow \Lambda_{c}^{+} \bar{\Lambda}_{c}^{-}$cross section near threshold. Phys. Rev. Lett. 2018, 120, 132001. [CrossRef] [PubMed]

37. Dobbs, S.; Seth, K.K.; Tomaradze, A.; Xiao, T.; Bonvicini, G. First measurements of timelike form factors of the hyperons, $\Lambda, \Sigma^{0}, \Sigma^{+}, \Xi^{0}, \Xi^{-}$, and $\Omega^{-}$, and evidence of diquark correlations. Phys. Lett. B 2014, 739, 90. [CrossRef]

38. Dobbs, S.; Seth, K.K.; Tomaradze, A.; Xiao, T.; Bonvicini, G. Hyperon form factors and diquark correlations. Phys. Rev. D 2017, 96, 092004. [CrossRef]

39. Zyla, P.A.; Barnett, R.M.; Beringer, J.; Dahl, O.; Dwyer, D.A.; Groom, D.E.; Lin, C.-J.; Lugovsky, K.S.; Pianori, E.; Robinson, D.J.; et al. Review of Particle Physics. Prog. Theor. Exp. Phys. 2020, 2020, 083 C01.

40. Seth, K.K.; Dobbs, S.; Metreveli, Z.; Tomaradze, A.; Xiao, T.; Bonvicini, G. Electromagnetic Structure of the Proton, Pion, and Kaon by High-Precision Form Factor Measurements at Large Timelike Momentum Transfers. Phys. Rev. Lett. 2013, 110, 022002. [CrossRef] [PubMed]

41. Brodsky, S.J.; Farrar, G.R. Scaling laws at large transverse momentum. Phys. Rev. Lett. 1973, 31, 1153. [CrossRef]

42. Ablikim, M.; Achasov, M.N.; Adlarson, P.; Ahmed, S.; Albrecht, M.; Amoroso, A.; An, Q.; Bai, Y.; Bakina, O.; Baldini Ferroli, R.; et al. Measurements of $\Sigma^{+}$and $\Sigma^{-}$timelike electromagnetic form factors for center-of-mass energies from 2.3864 to $3.0200 \mathrm{GeV}$. Phys. Lett. B 2021, 814, 136110. [CrossRef]

43. Ablikim, M.; Achasov, M.N.; Adlarson, P.; Ahmed, S.; Albrecht, M.; Amoroso, A.; An, Q.; Bai, Y.; Bakina, O.; Baldini Ferroli, R.; et al. Measurement of the $e^{+} e^{-} \rightarrow \Sigma^{0} \bar{\Sigma}^{0}$ cross sections at center-of-mass energies from 2.3864 to 3.0200 GeV. arXiv 2021, arXiv:2110.04510v1.

44. Chernyak, V.L.; Zhitnitsky, A.R. Asymptotic behavior of exclusive processes in QCD. Phys. Rept. 1984, 112, 173. [CrossRef]

45. Chernyak, V.L.; Ogloblin, A.A.; Zhitnitsky, I.R. Wave Functions of Octet Baryons. Z. Phys. C 1989, 42, 569. [CrossRef]

46. Ablikim, M.; Achasov, M. N.; Ahmed, S.; Ai, X. C.; Albayrak, O.; Albrecht, M.; Ambrose, D. J.; Amoroso, A.; An, F. F.; An, Q.; et al. Observation of a cross-section enhancement near mass threshold in $e^{+} e^{-} \rightarrow \Lambda \bar{\Lambda}$. Phys. Rev. D 2018, 97, 032013. [CrossRef]

47. Ablikim, M.; Achasov, M.N.; Adlarson, P.; Ahmed, S.; Albrecht, M.; Alekseev, M.; Amoroso, A.; An, F.F.; An, Q.; Bai, Y.; et al. Complete measurement of the $\Lambda$ electromagnetic form factors. Phys. Rev. Lett. 2019, 123, 122003. [CrossRef] [PubMed]

48. El-Bennich, B.; Lacombe, M.; Loiseau, B.; Wycech, S. Paris $N \bar{N}$ potential constrained by recent antiprotonic-atom data and $\bar{n} p$ total cross sections. Phys. Rev. C 2009, 79, 054001. [CrossRef] 
49. Baldini, R.; Pacetti, S.; Zallo, A.; Zichichi, A. Unexpected features of $e^{+} e^{-} \rightarrow p \bar{p}$ and $e^{+} e^{-} \rightarrow \Lambda \bar{\Lambda}$ cross sections near threshold. Eur. Phys. J. A 2009, 39, 315-321. [CrossRef]

50. Ferroli, R.B.; Pacetti, S.; Zallo, A. No Sommerfeld resummation factor in $e^{+} e^{-} \rightarrow p \bar{p}$. Eur. Phys. J. A 2012, 48, 33. [CrossRef]

51. Brodsky, S.J.; Hoang, A.H.; Kuhn, J.H.; Teubner, T. Angular distributions of massive quarks and leptons close to threshold. Phys. Lett. B 1995, 359, 355. [CrossRef]

52. Ablikim, M.; Achasov, M.N.; Adlarson, P.; Ahmed, S.; Albrecht, M.; Alekseev, M.; Amoroso, A.; An, F.F.; An, Q.; Bai, Y.; et al. Future physics program at BESIII. Chin. Phys. C 2020, 44, 040001. [CrossRef]

53. Zhou X. Experimental Program at Super Tau-Charm Facility. PoS (CHARM2020) 007. 2021. Available online: https://inspirehep. net/literature/1926635 (accessed on 15 November 2021).

54. Bondar, A.E. Project of a Super Charm-Tau factory at the Budker Institute of Nuclear Physics in Novosibirsk. Phys. Atom. Nucl. 2013, 76, 1072. [CrossRef]

55. Belias, A. FAIR status and the PANDA experiment. JINST 2020, 15, C10001. [CrossRef] 